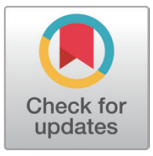

Received: Sep 10, 2020

Revised: Oct 22, 2020

Accepted: Oct 23, 2020

\#These authors contributed equally to this work.

*Corresponding author

Hyeun Bum Kim

Department of Animal Resources

Science, Dankook University, Cheonan

31116 , Korea.

Tel: +82-41-550-3653

E-mail: hbkim@dankook.ac.kr

Ju-Hoon Lee

Department of Food and Animal

Biotechnology, Department of

Agricultural Biotechnology, Center

for Food and Bioconvergence, Seoul

National University, Seoul 08826, Korea

Tel: +82-2-880-4854

E-mail: juhlee@snu.ac.kr

Copyright (c) 2021 Korean Society of Animal Sciences and Technology. This is an Open Access article distributed under the terms of the Creative Commons Attribution Non-Commercial License (http:// creativecommons.org/licenses/by$\mathrm{nc} / 4.0 /$ ) which permits unrestricted non-commercial use, distribution, and reproduction in any medium, provided the original work is properly cited.

\section{Complete genome sequence of Salmonella enterica strain K_SA184, multidrug resistance bacterium isolated from lamb (Ovis aries)}

\author{
Hyeri Kim ${ }^{1 \#}$, Jae Hyoung Cho ${ }^{1 \#}$, Jin Ho Cho ${ }^{2 \#}$, Minho Song ${ }^{3 \#}$, Hakdong Shin ${ }^{4}$, \\ Sheena Kim ${ }^{1}$, Eun Sol Kim ${ }^{1}$, Hyeun Bum Kim ${ }^{1 *}$ and Ju-Hoon Lee ${ }^{5 *}$ \\ ${ }^{1}$ Department of Animal Resources Science, Dankook University, Cheonan 31116, Korea \\ ${ }^{2}$ Division of Food and Animal Science, Chungbuk National University, Cheongju 28644, Korea \\ ${ }^{3}$ Division of Animal and Dairy Science, Chungnam National University, Daejeon 34134, Korea \\ ${ }^{4}$ Department of Food Science and Biotechnology, College of Life Science, Sejong University, Seoul \\ 05006, Korea \\ ${ }^{5}$ Department of Food and Animal Biotechnology, Department of Agricultural Biotechnology, Center for \\ Food and Bioconvergence, Seoul National University, Seoul 08826, Korea
}

\begin{abstract}
Salmonella enterica is a representative foodborne pathogen in the world. The S. enterica strain K_SA184 was isolated from the lamb (Ovis aries), which was collected from a local traditional market in South Korea. In this study, the S. enterica strain K_SA184 was sequenced using PacBio RS II and Illumina NextSeq 500 platforms. The final complete genome of the S. enterica strain K_SA184 consist of one circular chromosome $(4,725,087$ bp) with $52.3 \%$ of guanine + cytosine $(G+C)$ content, 4,363 of coding sequence (CDS), 85 of tRNA, and 22 of rRNA genes. The S. enterica strain K_SA184 genome includes encoding virulence genes, such as Type III secretion systems and multidrug resistance related genes.

Keywords: Salmonella enterica K_SA184, Lamb (Ovis aries), Whole genome sequencing, Antimicrobial resistance, Type III secretion systems
\end{abstract}

\section{INTRODUCTION}

Salmonella is a representative foodborne pathogen which is the most commonly identified in poultry, eggs and dairy products. The most common symptom of salmonella infection is gastroenteritis, follow by bacteremia and enteric fever. Most forms of poultry meat, pork, and beef are the main sources responsible for salmonella infection [1] because the contamination of the organ and carcass with salmonella easily occurs during the slaughtering process of the food animals at abattoirs [2].

The Salmonella enterica strain K_SA184 was isolated from a lamb (Ovis aries) purchased from the local traditional market in Suwon, Gyeonggi-do, Korea. The S. enterica strain K_SA184 was streaked to xylose lysine tergitol 4 (XLT4) agar and incubated at $37^{\circ} \mathrm{C}$ for $24 \mathrm{~h}$. The suspected colony in XLT4 agar was inoculated into Luria-Bertani (LB) broth and incubated at $37^{\circ} \mathrm{C}$ for $24 \mathrm{~h}$. To analyze the complete genome, the S. enterica strain K_SA184 was sequenced by PacBio RS II (Pacific Biosciences, Menlo 
ORCID

Hyeri Kim

https://orcid.org/0000-0002-6560-2390

Jae Hyoung Cho

https://orcid.org/0000-0002-1128-3451

Jin Ho Cho

https://orcid.org/0000-0001-7151-0778

Minho Song

https://orcid.org/0000-0002-4515-5212

Hakdong Shin

https://orcid.org/0000-0001-7615-9809

Sheena Kim

https://orcid.org/0000-0002-5410-1347

Eun Sol Kim

https://orcid.org/0000-0001-8801-421X

Hyeun Bum Kim

https://orcid.org/0000-0003-1366-6090

Ju-Hoon Lee

https://orcid.org/0000-0003-0405-7621

Competing interests

No potential conflict of interest relevant to

this article was reported.

\section{Funding sources}

The present study was supported by the research fund (19162MFDS037) from the Ministry of Food and Drug Safety, Korea, and by the University Innovation Support Program through the National Research Foundation of Korea (NRF) funded by the Ministry of Education (Dankook University 2019).

\section{Acknowledgements}

We thank Mo Re Kim (Brandeis University,

MA, USA) for the English grammar

corrections.

Availability of data and material The complete genome sequences of Salmonella enterica K SA184 were deposited in GeneBank under the accession numbers CP061159.1. The BioSample accession number is SAMN15891899, and BioProject accession number is PRJNA658857.

\section{Authors' contributions}

Conceptualization: Cho Jin Ho, Song M, Kim $\mathrm{HB}$, Lee JH.

Data curation: Kim H, Shin H, Kim S, Kim ES.

Formal analysis: Kim H, Shin H, Kim S, Kim ES.

Methodology: Kim H, Cho Jae Hyoung, Song M. Software: Kim H, Cho Jae Hyoung, Song M. Validation: Kim H, Shin H, Kim S, Kim ES

Investigation: Kim H, Cho Jae Hyoung, Song M, Kim HB, Lee JH.

Writing - original draft: Kim $\mathrm{H}$, Cho Jin $\mathrm{Ho}$ Song M, Kim HB, Lee JH.

Writing - review \& editing: Kim $\mathrm{H}$, Cho Jin $\mathrm{Ho}$, Song M, Kim HB, Lee JH.

Ethics approval and consent to participate This article does not require IRB/IACUC approval because there are no human and animal participants.
Park, CA, USA) at Insilicogen (Yong-in, Korea) and Illumina NextSeq 500 (Illumina, San Diego, CA, USA) platform at LabGenomics (Seongnam, Korea) [3]. The genomic DNA of S. enterica K_ SA184 for PacBio and Illumina sequencing were extracted using the MagAttract HMW DNA Kit (QIAGEN), and NucleoSpin ${ }^{\circledR}$ Microbial DNA kit (TAKARA) according to the manufacturer's instructions. Library preparation was conducted using SMRTbell ${ }^{\mathrm{TM}}$ Template Prep Kit 1.0 for Pacbio (Pacific Biosciences) and TruSeq DNA Sample Preparation Kit for Illumina (Illumina) according to the manufacturer's instructions. PacBio sequencing yielded 1,474,738,487 base pairs and 190,304 long reads after filtering, and 5,513,948 paired-end reads with 832,606,148 bp was obtained with Illumina sequencing. De novo assemble was conducted using the hierarchical genome assembly process (HGAP v2.3.0) workflow and polished using Quiver. Subsequently, Illumina NextSeq reads were aligned to the PacBio RSII assembly using Burrows-Wheeler Aligner (BWA)-MEM v0.7.17-r1188, and the errors were corrected by using Pilon version 1.23 [4,5]. The quality of genome assembly and the validation of the final genome were assessed by using Quality Assessment Tool for Genome Assemblies (QUAST) v5.0.2 and Benchmarking Universal SingleCopy Orthologs (BUSCO) v3.0.2 [6,7].

Open reading frames (ORFs) and RNA genes of S. enterica strain K_SA184 were predicted and functionally annotated by rapid prokaryotic genome annotation (PROKKA) v1.14.5 and Rapid Annotation using Subsystem Technology (RAST) v2.0. The functional categorization and classification of all predicted ORFs were conducted using the RAST server-based SEED viewer and Clusters of Orthologous Groups (COG) - based EggNOG. The putative virulence factors and Antimicrobial resistance were described using BLAST according to the Virulence Factor Database (VFDB) and antibiotic resistome surveillance with the comprehensive antibiotic resistance (CARD) $[8,9]$. The whole genome of $S$. enterica strain K_SA184 is composed of one circular chromosome $(4,725,087 \mathrm{bp})$ with $52.3 \%$ of guanine + cytosine $(\mathrm{G}+\mathrm{C})$ content, 4,363 of coding sequence (CDS), 85 of tRNA, and 22 of rRNA genes.

The complete genome of the S. enterica strain K_SA184 contains the virulence genes encoding Salmonella pathogenicity island $1 \& 2$ Type III secretion systems which serve several pathogenic functions in killing of macrophages and in interference with immune responses as reported by others [10]. Furthermore, the S. enterica strain K_SA184 also possesses multidrug resistance coding genes which are associated with a variety of drugs resistance Efflux Pumps (mdtk) and Resistance

\section{Table 1. Genome features of Salmonlla enterica strain K_SA184}

\begin{tabular}{|c|c|}
\hline Property & Term \\
\hline Libraries used & $\begin{array}{c}\text { PacBio SMRTbell }{ }^{\mathrm{TM}} \text { library } \\
\text { TruSeq DNA Sample Preparation Kit }\end{array}$ \\
\hline Sequencing platforms & $\begin{array}{l}\text { PacBio RS II sequencer } \\
\text { Illumina NextSeq } 500\end{array}$ \\
\hline Assemblers & PacBio SMRT analysis v2.3.0 HGAP.3 \\
\hline Annotation method & PROKKA v1.14.5 and RAST v2.0 \\
\hline Average genome coverage & $159 x$ \\
\hline Chromosome length (bp) & $4,725,087$ bp \\
\hline No. of contigs & 1 \\
\hline guanine + cytosine $(G+C)$ content $(\%)$ & 52.3 \\
\hline Protein-coding genes (CDSs) & 4,363 \\
\hline rRNA genes & 22 \\
\hline tRNA genes & 85 \\
\hline Plasmids & 0 \\
\hline Genbank accession No. & СР061159.1 \\
\hline
\end{tabular}




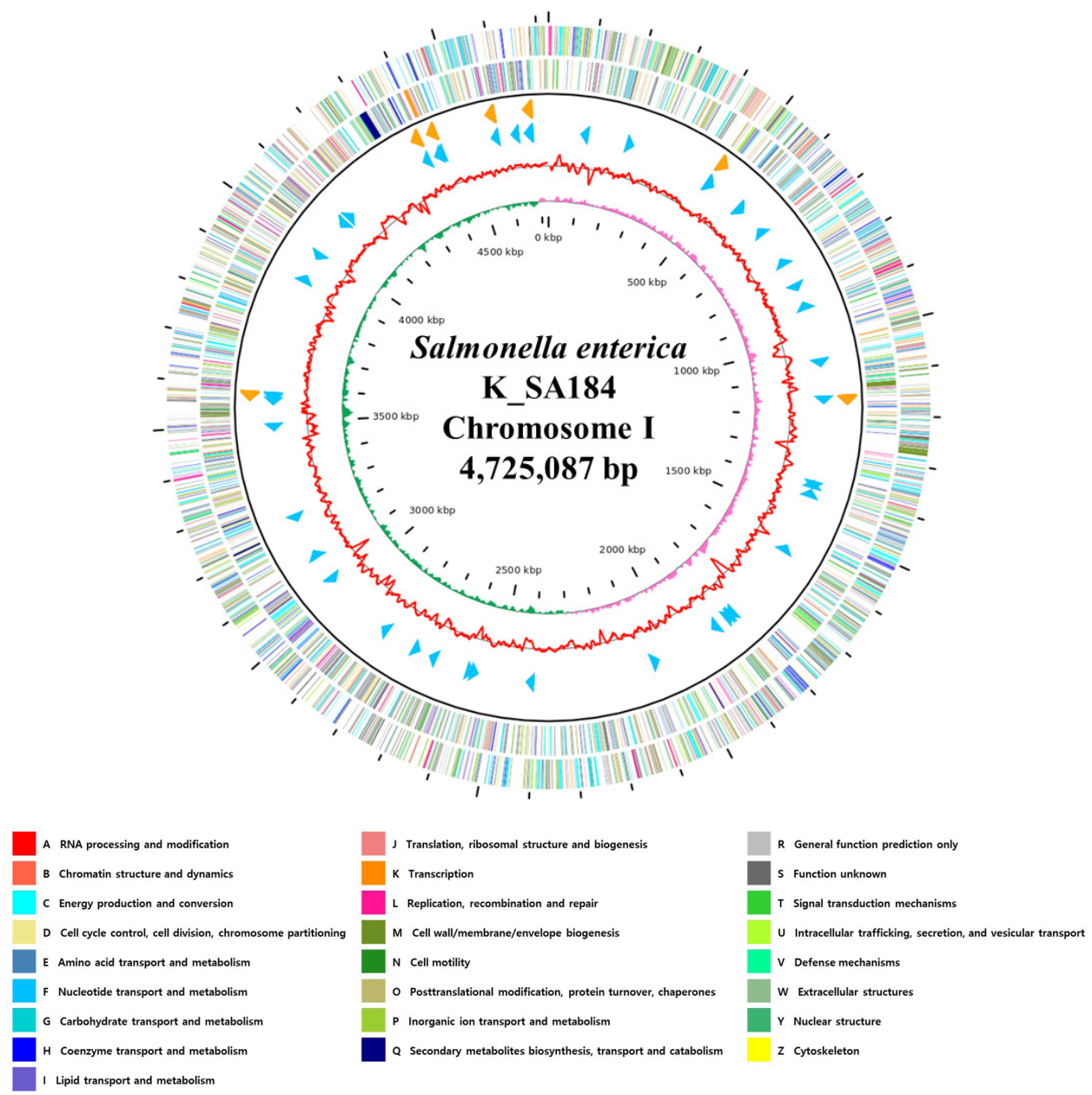

Fig. 1. Genome map of Salmonella enterica strain K_SA184. The outer circle denotes the locations of all annotated open reading frames (ORFs), and the inner circle with the red denotes guanine + cytosine (GC) content. Pink, and green peaks denote GC skew. The orange arrows denote rRNAs, and the sky blue arrows denote the tRNA operons. All annotated ORFs are colored differently based on the Clusters of Orthologous Groups (COG) assignments.

to fluoroquinolones, such as, cephalosporins (AmpC), and fluoroquinolones (Par, Gyr). We summarized the general properties of the S. enterica strain K_SA184's complete genome in Fig. 1 and Table 1. The further in-vivo studies using S. enterica strain K_SA184 will help us to decipher the potential roles of the virulence genes in the pathogenesis.

\section{REFERENCES}

1. Guevarra RB, Lee JH, Lee SH, Seok MJ, Kim DW, Kang BN, et al. Piglet gut microbial shifts early in life: causes and effects. J Anim Sci Biotechnol. 2019;10:1. https://doi.org/10.1186/ s40104-018-0308-3

2. Eng SK, Pusparajah P, Ab Mutalib NS, Ser HL, Chan KG, Lee LH. Salmonella: a review on pathogenesis, epidemiology and antibiotic resistance. Front Life Sci. 2015;8:284-93.

3. Ryu S, Shin D, Heo J, Jeong SY, Jeong DY, Yun B, et al. Complete chromosome and plasmid sequences of Staphylococcus aureus strain JDFM SA01, isolated from a milk filter in Korean dairy farm.J Anim Sci Technol. 2020;62:423-6. https://doi.org/10.5187/jast.2020.62.3.423 
4. Li H. Aligning sequence reads, clone sequences and assembly contigs with BWA-MEM. arXiv:13033997v1 [preprint]. 2013 [cited 2020 Aug 9]. https://arxiv.org/abs/1303.3997

5. Walker BJ, Abeel T, Shea T, Priest M, Abouelliel A, Sakthikumar S, et al. Pilon: an integrated tool for comprehensive microbial variant detection and genome assembly improvement. PLOS ONE. 2014;9:e112963. https://doi.org/10.1371/journal.pone.0112963

6. Simão FA, Waterhouse RM, Ioannidis P, Kriventseva EV, Zdobnov EM. BUSCO: assessing genome assembly and annotation completeness with single-copy orthologs. Bioinformatics. 2015;31:3210-2. https://doi.org/10.1093/bioinformatics/btv351

7. Gurevich A, Saveliev V, Vyahhi N, Tesler G. QUAST: quality assessment tool for genome assemblies. Bioinformatics. 2013;29:1072-5. https://doi.org/10.1093/bioinformatics/btt086

8. Alcock BP, Raphenya AR, Lau TTY, Tsang KK, Bouchard M, Edalatmand A, et al. CARD 2020: antibiotic resistome surveillance with the comprehensive antibiotic resistance database. Nucleic Acids Res. 2020;48:D517-25. https://doi.org/10.1093/nar/gkz935

9. Liu B, Zheng D, Jin Q, Chen L, Yang J. VFDB 2019: a comparative pathogenomic platform with an interactive web interface. Nucleic Acids Res. 2019;47:D687-92. https://doi. org/10.1093/nar/gky1080

10. Galán JE. Common themes in the design and function of bacterial effectors. Cell Host Microbe. 2009;5:571-9. https://doi.org/10.1016/j.chom.2009.04.008 